\title{
COMPUTATIONAL AND EXPERIMENTAL VERIFICATION OF THE EQUIVALENT PERMEABILITY OF THE STEP-LAP JOINTS OF TRANSFORMER CORES
}

\author{
N. Hihat*, E. Napieralska-Juszczak*, J. Ph. Lecointe*, J. K. Sykulski ${ }^{\dagger}$ \\ *LSEE, Universite d'Artois, 62400 Bethune, Technoparc Futura , France \\ ${ }^{\dagger}$ School of Electronics and Computer Science, University of Southampton, SO17 1BJ, United Kingdom \\ ewa.napieralskajuszczak@univ-artois.fr, jks@soton.ac.uk
}

Keywords: Step-lap joints, anisotropic laminations, equivalent magnetic characteristics.

\section{Abstract \\ The paper develops an efficient computational method for establishing equivalent characteristics of magnetic joints of transformer cores, with special emphasis on step-lap design. This enables comparative analysis of designs without the need for difficult measurements.}

\section{Introduction}

This paper introduces a method for computation of equivalent magnetic characteristics for structures built from anisotropic lamination sheets [1, 2]. A twodimensional formulation is employed as the magnetic flux density component perpendicular to the surface of the sheet $\left(B_{z}\right)$ is negligible: if no air-gaps are present, and for the resultant $B$ of $1.8 \mathrm{~T}$, this value is less than $0.01 \mathrm{~T}$, if air-gaps exist it will be around 0.06T [3-5]. A sample of dimensions $\delta x \times \delta y \times \delta z \quad(50 \times 50 \times 5 \mathrm{~mm})$ was analysed experimentally and computationally and the flux density components may be found as

$$
B_{i x}=\frac{1}{2} \frac{\phi_{i x}^{\prime}+\phi_{i x}^{\prime \prime}}{\delta y \delta z} ; \quad B_{i y}=\frac{1}{2} \frac{\phi_{i y}^{\prime}+\phi_{i y}^{\prime \prime}}{\delta x \delta z},
$$

where $i=1,2, \ldots, n$ denotes the structure's layer, $\phi_{i x}^{\prime}$ is the flux 'in' through the surface $y z, \phi_{i x}$ is the flux 'out', $\phi_{i y}^{\prime}$ and $\phi_{i y}^{\prime \prime}$ are similar fluxes through the surface $x z$. In order to model the real 3D situation approximately as a 2D problem, it has been assumed that the structure is built from an equivalent material giving identical field distribution as the original. The actual field distribution is a result of the system's tendency to achieve a minimum of magnetic energy, which may be expressed as

$$
W_{\mu \min }=\sum_{i=1}^{n} W_{\mu i}\left(\mu_{i}, \vec{B}_{i}\right)
$$

where $\mu_{l}$ is the permeability, and $\vec{B}_{i}$ the induction, in individual laminations. The equivalent permeability at any point of the core is then a function of the resultant flux density and the minimal field energy $\mu\left(\vec{B}_{e q}, W_{\mu \min }\right)$.

\section{Equivalent characteristics for step-lap joints}

The step-lap joint uses vertical displacements of sheets in consecutive cycles of core assembly [5]. In this paper we have assumed 5 cycles resulting in 4 'steps'. The purpose is to reduce the overlap of the joint and spreading of the air-gaps. This, in turn, reduces iron loss by about $5 \%$ in comparison with the conventional $45^{\circ}$ and $90^{\circ}$ designs [6]. Moreover, this structure is less sensitive to the inaccuracies of the sheet assembly. Denoting by $x$ the yoke's laminations and by $y$ the column's ones, the joints may be made of 4 steps of unequal numbers of sheets following the pattern: $1 x-4 y$, $2 x-3 y, 3 x-2 y$ and $4 x-1 y$. The width of the step in our case is $3 \mathrm{~mm}$ while the air-gap is $0.25 \mathrm{~mm}$. There are $n_{1}$ sheets in the yoke with flux density $\vec{B}_{1}$ and $n_{2}$ sheets in the column with density $\vec{B}_{2}$, the air-gap flux density is $\vec{B}_{0}$ with $n_{0}$ sheets separated by the gap. The flux density of the equivalent structure may then be defined as

$$
\left(n_{1}+n_{2}+n_{3}\right) \vec{B}=n_{1} \vec{B}_{1}+n_{2} \vec{B}_{2}+n_{0} \vec{B}_{0}
$$

The energy stored in the virtual gap (Fig. 1) in the joint

$$
W_{\mu}=\frac{1}{2} V_{s h}\left(n_{0} v_{0} B_{0}^{2}+n_{1} v_{1} B_{1}^{2}+n_{2} v_{2} B_{2}^{2}\right)
$$

where $v_{1}$ and $v_{2}$ are the reluctivities of the yoke and the column, respectively, while $v_{0}=1 / \mu_{0}$ and $V_{s h}$ is the lamination volume. The objective function in the minimisation process may be defined as

$$
f=n_{0} v_{0} B_{0}^{2}+n_{1} v_{1} B_{1}^{2}+n_{2} v_{2} B_{2}^{2}=\min
$$

subject to the constraint (3). The unknowns are the components of $\vec{B}_{1}$ and $\vec{B}_{2}$. A standard Hooke and Jeeves Direct Search Method has been used as it is known to be robust and insensitive to the starting point.

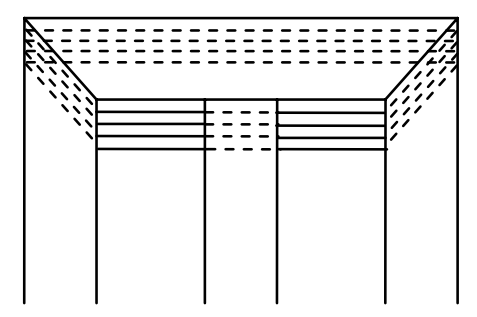

Fig. 1. Internal virtual gaps in the joint.

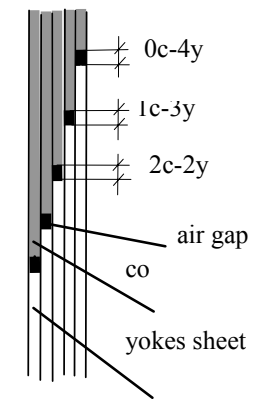

The starting values for $B_{1 x}, B_{1 y}, B_{2 x}$ and $B_{2 y}$ follow an initial assumption that magnetic flux goes entirely through the laminations avoiding the gaps. Moreover, for small values of induction the flux goes mainly along the rolling direction, whereas for larger values $\vec{B}_{1} \approx \vec{B}_{2}$ 
(Table. 1.) The equivalent reluctivity $v_{e q}$ of the homogenised replacement material representing the laminated system of the joint may be found by equalling the energies of the real and equivalent structures

$$
\frac{1}{2}\left(n_{0}+n_{1}+n_{2}\right) V_{s h} v_{e q} B_{e q}^{2}=\frac{1}{2} V_{s h}\left(n_{0} v_{0} B_{0}^{2}+n_{1} v_{1} B_{1}^{2}+n_{2} v_{2} B_{2}^{2}\right)
$$

\begin{tabular}{|l|l|l|l|l|l|l|l|}
\hline $\mathrm{B}$ & $\mathrm{B}_{\mathrm{ox}}$ & $\mathrm{B}_{\mathrm{oy}}$ & $\mathrm{B}_{1 \mathrm{x}}$ & $\mathrm{B}_{1 \mathrm{y}}$ & $\mathrm{B}_{2 \mathrm{x}}$ & $\mathrm{B}_{2 \mathrm{y}}$ & structure \\
\hline 0.5 & 0 & 0 & 0.44 & 0.44 & & & $4 \mathrm{c}-0 \mathrm{y}$ \\
\hline 0.5 & 0 & 0 & 0.59 & 0.24 & 0 & 1.06 & $3 \mathrm{c}-1 \mathrm{y}$ \\
\hline 0.5 & 0 & 0 & 0.88 & 0 & 0 & 0.88 & $2 \mathrm{c}-2 \mathrm{y}$ \\
\hline 1.5 & 0.06 & 0.07 & 1.31 & 1.3 & & & $4 \mathrm{c}-0 \mathrm{y}$ \\
\hline 1.5 & 0.02 & 0.05 & 1.63 & 1.13 & 0.4 & 1.87 & $3 \mathrm{c}-1 \mathrm{y}$ \\
\hline 1.5 & 0.03 & 0.03 & 1.77 & 0.87 & 0.86 & 1.76 & $2 \mathrm{c}-2 \mathrm{y}$ \\
\hline
\end{tabular}

Table. 1. Flux density vectors for small (0.5T) and large (1.5T) values of induction for the different structures (where 4c, 3c, 2c - number of column's sheets; and 0y, $1 \mathrm{y}, 2 \mathrm{y}$ - number of yoke's sheets of the joint).

\section{Results}

Equivalent $B / H$ curves have been computed for the structures containing the virtual gaps and are shown in Fig. 2. The curves are drawn for various angles of inclination of the resultant vector $B$ with respect to the horizontal line (coinciding with the yoke).

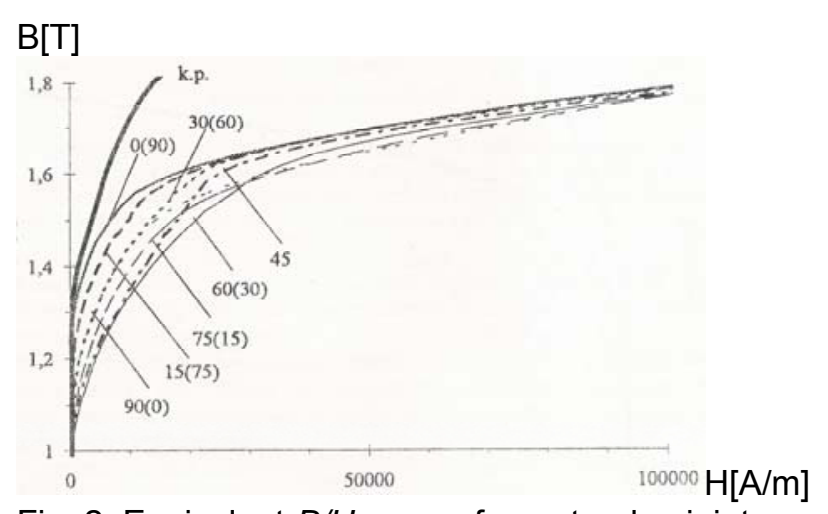

Fig. 2. Equivalent $B / H$ curves for a step-lap joint.

The following observations can be made.

1. The equivalent material has less anisotropy than the original (real) lamination sheets.

2. The less the difference between the volumes of the column and yoke laminations, the smaller the anisotropy of the equivalent material.

3. A parameter for the family of characteristics of the laminations, from which the joint is made, is the angle between the rolling and magnetisation directions, whereas for the equivalent material it is the angle between the resultant induction $\vec{B}_{e q}$ and the direction of rolling of laminations dominating the segment.

4. Up to about 1.5T, the equivalent characteristics (of the 'replacement' material) are similar to those of the original core lamination, while at field levels close to saturation they are significantly different.

\section{Experiments}

Measurements have been taken on a real sample, including resultant flux densities as well as those inside each layer. Narrow holes $(0.2 \mathrm{~mm})$ were drilled to install search coils which allow flux to be measured in the two relevant directions $\left(B_{x}\right.$ and $B_{y}$ at point $P$ ). The area of the search coils needs to be small to for the averaging not to distort the real distribution.

\section{Conclusions}

The ability to compute equivalent characteristics for different types of joints of the transformer core is important even in the early stages of the design. Measurements for the step-lap joint are difficult due to very small 'steps'. The proposed quasi-3D method is fast and sufficiently accurate for design purposes.

\section{Acknowlegment}

This work is supported by the program "Futurelec 8" supervised by the French National Center of Technological Research (CNRT) in Electrical Engineering. This program, which includes Thyssen Krupp, is sponsored by the region "Nord-Pas-deCalais", the French Ministry (FRT) and the European funds (FEDER).

\section{References}

[1] F. Loffler, T. Booth, H. Pfutzner, C. Bengtsson, K. Gramm. "Relevance of step-lap joints for magnetic characteristics of transformer cores", IEE Proc.Electr. Power Appl., 142(6), pp. 371-378, (1995)

[2] A. Mae, K. Harada, Y. Ishihara, T. Todaka. "A Study of Characteristic Analysis of the ThreePhase Transformer With Step-Lap Wound-Core", IEEE Trans. on Magn., 38(2), pp. 829-832, (2002)

[3] P. Hahne, D. Rainer, B. Rieth, T. Weiland. "Determination of anisotropic equivalent conductivity of laminated cores for numerical computation", IEEE Trans. Magn., 32(3), pp. 11841187, (1996)

[4] M. A. Jones, A. J. Moses, J. E. Thompson. "Flux distribution and power loss in the mitred overlap joint and power transformer cores." IEEE Trans on Magn., 6(2), pp. 114-122, (1973)

[5] G. Y. Xiao, A. J. Moses, F. Anayi. "Normal Flux Distribution in a Three-Phase Transformer Core Under Sinusoidal and PWM Excitation", IEEE Trans. on Magn., 43(6), pp. 2660-2662, (2007)

[6] J. C. Olivares, Y. Liu, J. M. Canedo, R. E. Perez, J. Driesen, P. Moreno. "Reducing Losses in Distribution Transformers", IEEE Trans. on Power Del., 18(3), pp. 821-827, (2003) 